\title{
Visual-based Crack Detection and Skeleton Extraction of Cement Surface
}

\author{
Du Jiang ${ }^{1}$, Gongfa Li ${ }^{1,2,3}$, Ying Sun ${ }^{4}$, Jianyi Kong ${ }^{1}$, Bo Tao ${ }^{2}$, Dalin Zhou ${ }^{5}$, Disi \\ $\mathrm{Chen}^{5}$ and Zhaojie $\mathrm{Ju}^{5}$ \\ ${ }^{1}$ Key Laboratory of Metallurgical Equipment and Control Technology, Ministry \\ of Education, Wuhan University of Science and Technology, Wuhan, 430081, \\ China \\ 2 Institute of Precision Manufacturing, Wuhan University of Science and Tech- \\ nology, Wuhan University of Science and Technology, Wuhan, 430081, China \\ 3.Research Center for Biomimetic Robot and Intelligent Measurement and Con- \\ trol, Wuhan University of Science and Technology, Wuhan, 430081, China \\ 4. Hubei Key Laboratory of Mechanical Transmission and Manufacturing Engineer- \\ ing, Wuhan University of Science and Technology, Wuhan, 430081, China \\ 5. School of Computing, University of Portsmouth P01 3HE, UK \\ Du Jiang: $1439078161 @ q q . c o m$ \\ Gongfa Li: ligongfa@wust.edu.cn \\ Ying Sun: sunying65@wust.edu.cn \\ Jianyi Kong: 15697188659@wo.com.cn \\ Bo Tao: taoboq@wust.edu.cn \\ Dalin Zhou: Dalin. zhou@port. ac.uk \\ Disi Chen: chendisi@foxmail.com \\ Zhaojie Ju: zhaojie.ju@port.ac.uk
}

\begin{abstract}
In order to realize the design of vision-based cement crack repair robot, it is necessary to accurately recognize and extract features of cracks. In this paper, three kinds of typical crack are selected to study, which are fine crack, reticulated crack and dark crack. Firstly, image filtering and image enhancement are used to pre-process the collected image to reduce the influence of noise on detection and enhance the contrast between image background and crack area. Then, the multi-scale morphological operation is applied to extract the fracture edge features effectively. The experimental results show that the proposed edge regions are obviously different from the background regions. Furthermore, by calculating and selecting the area of the largest connected area, the noise can be eliminated to the greatest extent. Finally, the traditional skeleton extraction algorithm is improved to eliminate the number of burrs in the traditional skeleton algorithm. By remapping the cracks images to color images, it can be found that the crack recognition and skeleton extraction meet the requirements, which can provide corresponding technical support for the navigation design of the crack repair robot.
\end{abstract}

Keywords: Crack recognization, Multi-scale morphology, Skeletonization. 


\section{Introduction}

With the national investment in infrastructure, China's urban underground pipeline network has been greatly developed and upgraded. With the increasing complexity of urban underground space and pavement construction, the maintenance and management of underground pipeline network has become more and more important. At present, the underground pipelines are mainly maintained through manual work when there is some problems such as burst, leak and so on, which greatly the increase the upkeep. Generally, if the sewage pipeline can be treated in time before the problem is complicated, it can effectively reduce the loss. Because of the special environment of underground sewage pipeline, it is difficult to detect and repair it in time. With the development of robotics technology, it brings new methods and solutions to solve this problem. Currently, there are mature products on the market to capture video of the environment of pipeline, which saves the cost of manpower and equipment needed for pipeline detection, however, their level of automation needs to be further replaced With the development of computer hardware and digital image processing technology, vision-based target location and detection technology have made great progress. Because of its accurate recognition and location, it provides a safe and efficient navigation means for the pipeline crack detection and repair robot based on digital image.

Visually, the enhancement and location of crack image on cement surface belongs to linear target detection while it has some unique characteristics, such as low contrast, intermittent, bifurcation and background noise. There are many research results on crack detection, such as threshold segmentation, edge detection, wavelet transform and so on [1]. Hyun-Seok et al. [2] used neural network to detect cracks, which improved the accuracy of crack detection. Shu et al. [3] provided an improved contour algorithm for crack detection. Petrik et al. [4] applied fuzzy idea to recognize crack. However, due to the poor shooting environment in the pipeline, the quality of the collected images will be poor, leading to the inability of the automatic detection algorithm. Therefore, it is necessary to add some image pre-processing before crack recognition to improve the quality of the images and reduce the interference of background noise. Image pre-processing is generally used as a pre-processing based on visual recognition whose main purpose is to highlight the features of the detected object and the region of interest, eliminate and reduce the interference of noise, such as image enhancement, image de-noising and edge feature extraction. Generally, the crack image will be disturbed by the environment. At the same time, because of the uncertainty of the crack and the uncertainty of the environment, it is difficult for the collected image to meet the requirements of detection. As a result, it is often necessary to add image preprocessing before realizing vision-based target recognition and detection.

The main purpose of our research is to study the crack detection on cement surface. Based on this, the information characteristics of crack skeleton need to be extracted, to provide the corresponding reference for the trajectory generation of automatic repair robot. In this paper, multi-scale morphological calculation and skeleton algorithm are mainly used to identify and extract the skeleton of multiple cracks. The proposed method further improves the accuracy of crack detection, and the experimental results 
show that it is helpful to the design of pipeline crack repair robot. Three typical fracture images are selected for study, as shown in Figure 1.

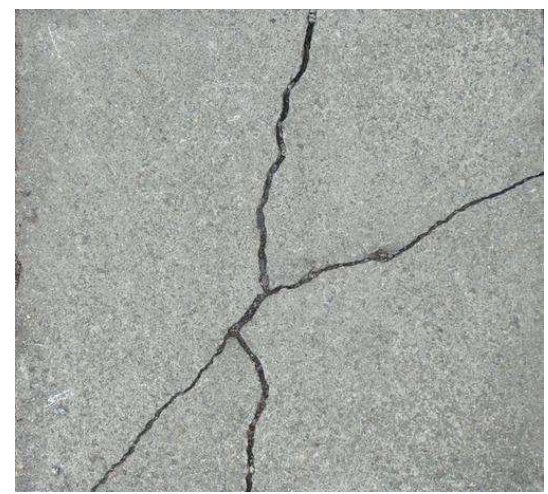

(a)

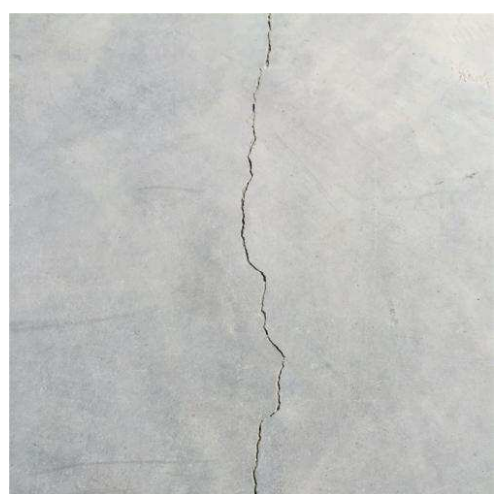

(b)

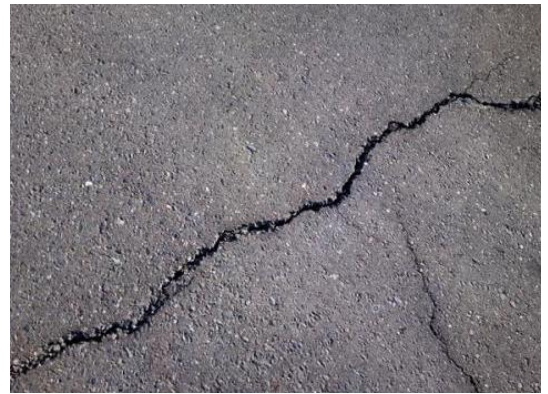

(c)

Fig. 1. Original image. (a): mesh crack, (b): fine crack, (c) dark crack

\section{$2 \quad$ Image preprocessing}

\subsection{Image filtering}

In order to improve the image quality and increase the accuracy of target detection and judgment, it is necessary to filter and de-noise the image by means of image processing [5]. In which, the commonly used filtering methods are Gauss filtering, mean filtering and median filtering. Because crack images often contain a large amount of particle noise, the median filtering method is used [6]. Median filtering is a kind of non-linear filtering method, which is simple and efficient. It can effectively remove impulse noise and protect edge information. Therefore, this paper uses median filter and square template to eliminate most of the noise in the original image and highlight the information of crack edge, which is conducive to the subsequent extraction of crack features. The effect of middle finger filtering is displayed on Figure 2. 


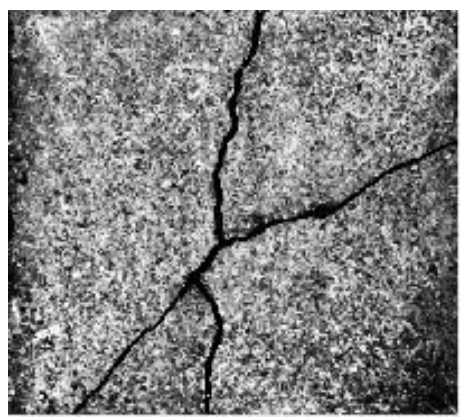

Fig. 2. Median filtering effect

\subsection{Image enhancement}

Pipeline image acquisition environment is generally complex, and it is easy to affect the quality of the image. The collected image may have a whole dark or low contrast image just like Fig.3(c), which will lead to difficulties in subsequent crack detection and feature extraction so it is necessary to use image enhancement technology to ameliorate the corresponding situation to a certain extent [7-8].

Histogram, as a statistical tool, can display the specific information of contrast to a certain extent. The gray histogram of the image shows the frequency of different gray levels in the gray level type of the image. Histogram equalization mainly uses gray histogram to adjust the image contrast, so as to enhance the image. With transformation, the histogram of the original image is transformed from a small gray scale to a uniform distribution in a larger gray scale, so as to enhance the overall contrast of the image.

Crack image area usually belongs to the gray-scale region with darker color, and the background area is brighter. However, due to the influence of environment, it is difficult to form obvious differences between the background area and the fracture area. Thus, histogram equalization can effectively enhance the contrast of images and highlight the difference between background and cracks. As can be seen from Fig. 3, the fracture area is obviously enhanced.

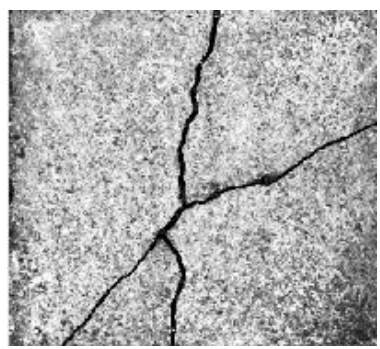

Fig. 3. Image enhancement effect 


\section{Extraction of cement crack contour based on multi-scale morphology}

\subsection{Principles of multiscale morphology}

Mathematical morphological image processing is based on set theory [9-10]. Morphological transformation of images is essentially a set-oriented processing process. Morphological operations can be used to represent the shape of objects, whose shape determines the shape information of objects matched by it. Therefore, the morphological operation of the image is to calculate the structural elements in the image and get the processing result matrix. The basic morphological operations of mathematical morphological image processing are corrosion and expansion. Their combination can be called opening and closing operations. Its basic principles are as follows:

Expand:

$$
A \oplus B=\max _{(i, j)}[A(x-i, y-j)+B(i, j)]
$$

Corrosion:

$$
A \Theta B=\min _{(i, j)}[A(x+i, y+j)-B(i, j)]
$$

Two combinations of expansion and corrosion operations can be obtained:

Opening operation:

$$
A \circ B=(A \Theta B) \oplus B
$$

Closing operation:

$$
A \bullet B=(A \oplus B) \Theta B
$$

In the above formulas, $A(x, y)$ represents the input image, and $B(x, y)$ represents structural elements. $\Theta$ and $\oplus$ represent Corrosion operation and expansion operation in morphology respectively.

Multiscale morphology is achieved by selecting the type and scale of structural elements, such as processing different images or dealing with different structural elements, which requires the selection of appropriate scale. Generally, with the increase of the scale of structural elements, the amount of computation will increase correspondingly, and even the geometric properties of images will be affected correspondingly, which will affect the final processing results. Selecting appropriate small-scale structural elements can improve the operation efficiency to a certain extent. Therefore, the edge detection of crack image is carried out by structural elements of different scales, and then the detected edge information is integrated by weighted fusion, so as to reduce the influence of noise in the image. 
According to the concepts of mathematical morphology, the definition of multiscale iterative filters is as follows:

$$
\psi(A)=\left(A \circ B_{1} \bullet B_{2}\right) \bullet B_{2} \circ B_{2}
$$

Multiscale structural elements are defined as:

$$
n B=B \oplus B \oplus \cdots \oplus B
$$

Among them, $n$ is a scale parameter.

Multi-scale edge detection algorithm:

$$
G_{i}^{n}=\left(A \circ n B_{i}\right) \oplus n B_{i}-\left(A \bullet n B_{i}\right) \Theta n B_{i}
$$

Multi-scale edge fusion algorithm:

$$
G A^{n}=\sum_{i=1}^{K} u_{i} G_{i}^{n}
$$

In which, $u_{i}$ is weighting coefficients for fusion of multi-scale edge detection images.

The set of positions where the values around the pixels change sharply is generally referred to as the image edge, which is one of the basic features of the image. Image edges generally exist between objects, background and regions, so edge extraction is one of the key steps in image segmentation. The image edges include the following kinds: step edge and roof edge. The gray value of the pixels around the step edge is obviously different, showing a step shape; the pixel value around the roof edge will have a peak value, showing the roof style.

\subsection{Result Analysis and Processing}

Through multi-scale edge extraction of three kinds of images, the results are as follows, shown on the Fig.4. By the way, the crack features of three types of crack images are effectively extracted, and the noise is effectively smoothed. From the analysis of the generated three-dimensional pseudo-color image (Fig.5), it can be found that there are obvious differences between the crack edge area and the background area, which provides a sufficient basis for the subsequent crack skeleton extraction. 


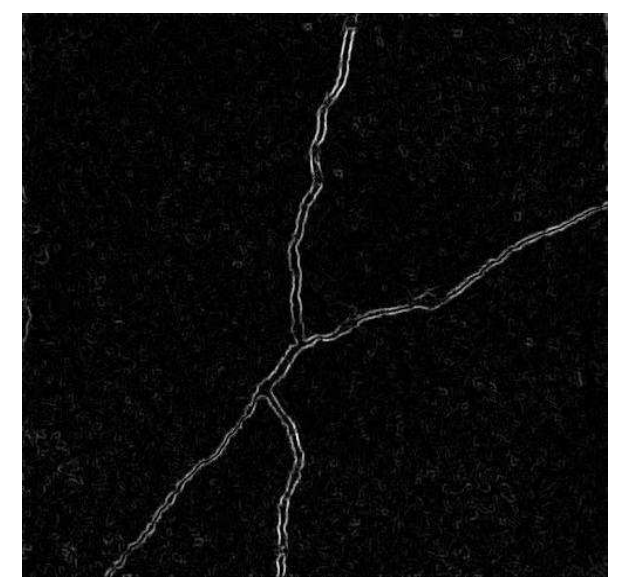

(a)

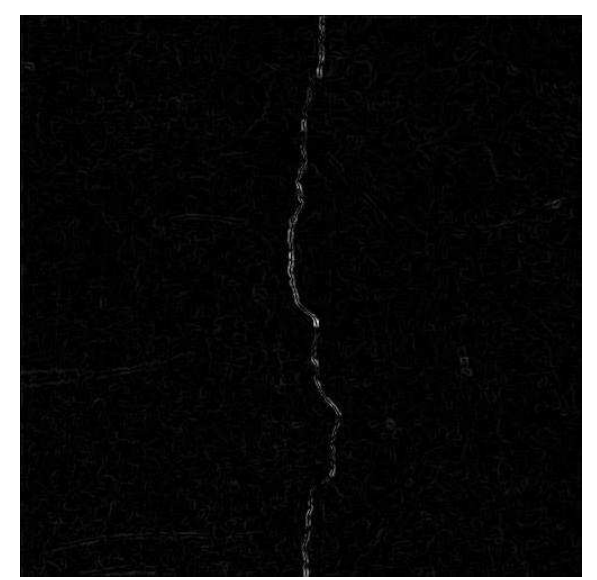

(b)

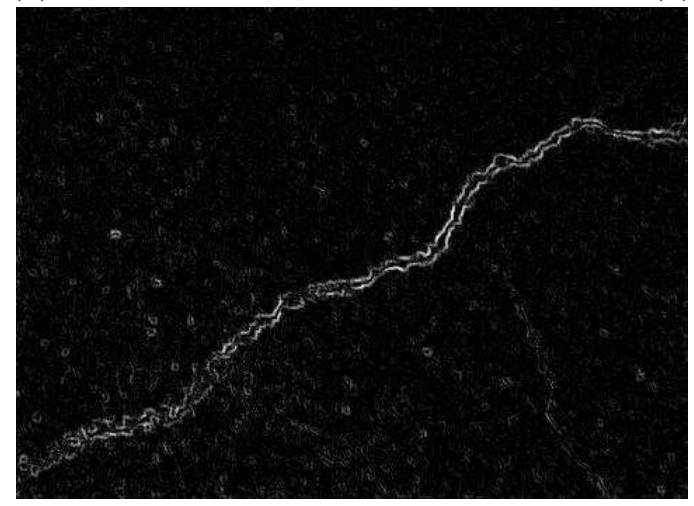

(c)

Fig. 4. Edge Contour Extraction.

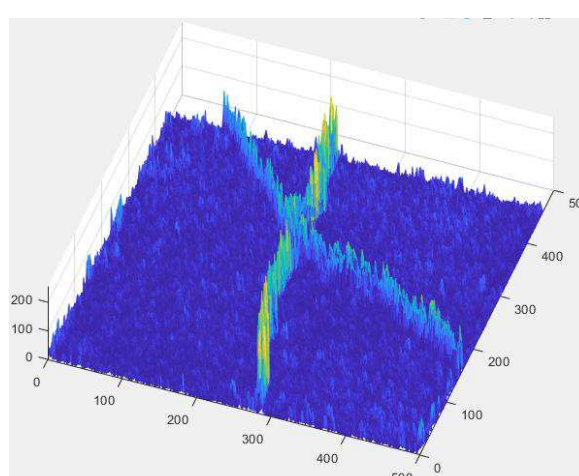

(a)

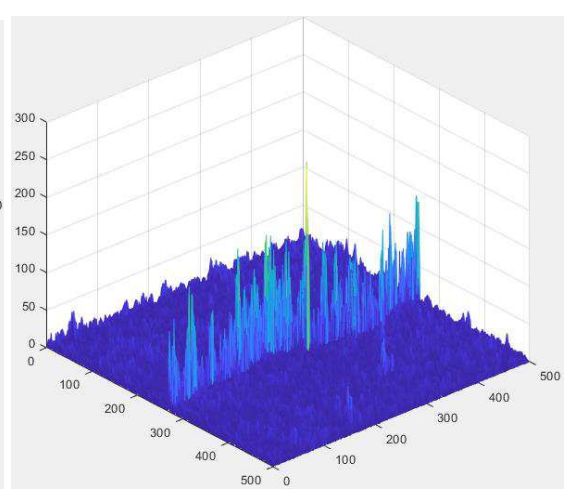

(b) 


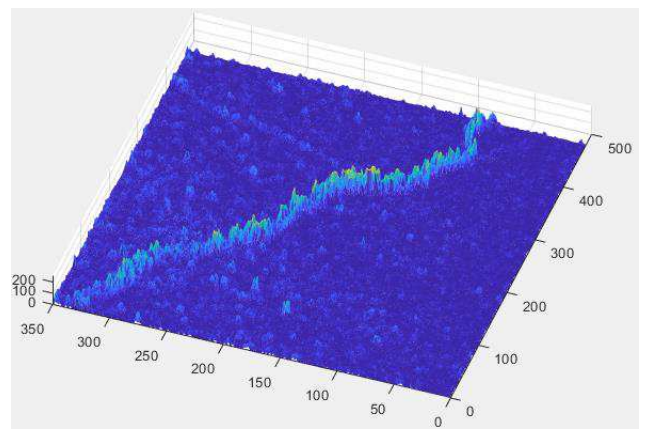

(c)

Fig. 5. Pseudo-color three-dimensional display.

\section{Skeleton extraction of cement cracks}

\subsection{Adaptive threshold image binarization}

The binarization of an image usually uses a threshold to segment the target area from the background. The pixels within the threshold are marked as 1, while the others belonging to the background are marked as 0 . Through the observation of the results of multi-scale edge extraction, we can find that there is a great difference in the gray value between the fracture target and the background area. Therefore, in order to segment fracture target and background to a greater extent, it is necessary to select appropriate threshold. There are two main methods for calculating thresholds: global thresholds and adaptive thresholds.

(1) Global threshold: The threshold is determined based on the histogram or gray spatial distribution of the image.

(2) Adaptive threshold: Based on the gray level change of the image pixel itself and its domain, threshold segmentation is carried out, and then the binary segmentation of gray image is realized.

The quality of crack image will change because of the change of shooting environment. Therefore, it is necessary to select a suitable threshold calculation method to achieve effective comparison between the edge area of crack and the background. This paper chooses an adaptive threshold calculation method. Fig.6 shows the binarization result of mesh crack with adaptive threshold. 


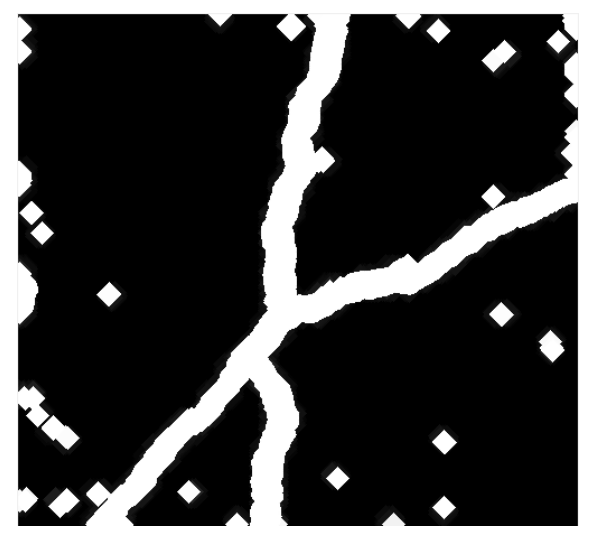

Fig. 6. The effect of adaptive image binarization

\subsection{Maximally connected region segmentation}

After binary segmentation, it can be found that there are still many noise points in the image. In order to further eliminate the noise in the background and highlight the crack area, it is necessary to expand the edge of the crack to form a larger connected area. Then, the connected area with the largest area is selected to eliminate the small noise points effectively.
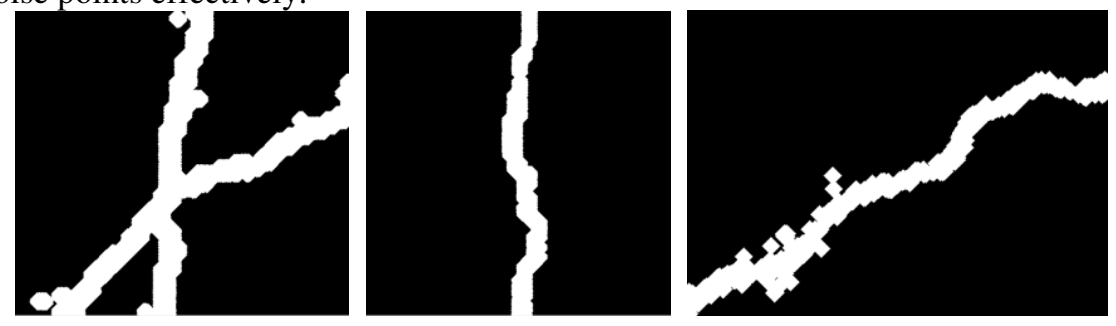

Fig. 7. The effect of maximally connected region segmentation

\subsection{Skeleton extraction}

In traditional skeleton extraction algorithms, the image is refined step by step by step through iterative etching operation, and skeleton extraction is finally realized [11-12]. But this will lead to a lot of burrs in the process of skeleton extraction, as shown in the Figure 8 . The existence of burrs interferes greatly with the trajectory generation of the robot. Therefore, it is necessary to deal with these burrs further. In this paper, a simple skeleton extraction algorithm is proposed. The skeleton extraction is realized by calculating the mean value of the crack area.

The flow chart of the algorithm is as follows:

(1) Judging the direction of cracks in the image area by row/column projection.

(2) Traversing the image with row/column benchmark to find the index value marked as 1 . 
(3) By averaging the index value, the image is taken as the skeleton point of the base row/column.

(4) The skeleton is formed by recording these skeleton points.

The experimental structure shows that this method can effectively remove skeleton burrs. However, there are still some shortcomings, such as the inaccurate judgment of traversal by rows and columns when extracting mesh cracks, which leads to the inaccurate average index value, especially the index value of mesh endpoints. By projecting the skeleton information of the crack onto the color image (Fig.10), it can be found that the method adopted in this paper can basically realize the marking of the crack on the cement surface.
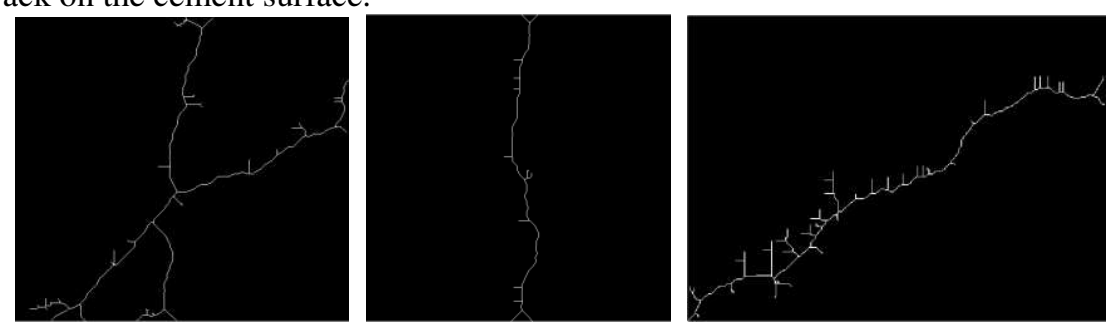

Fig. 8. Skeleton obtained by traditional skeleton algorithm.
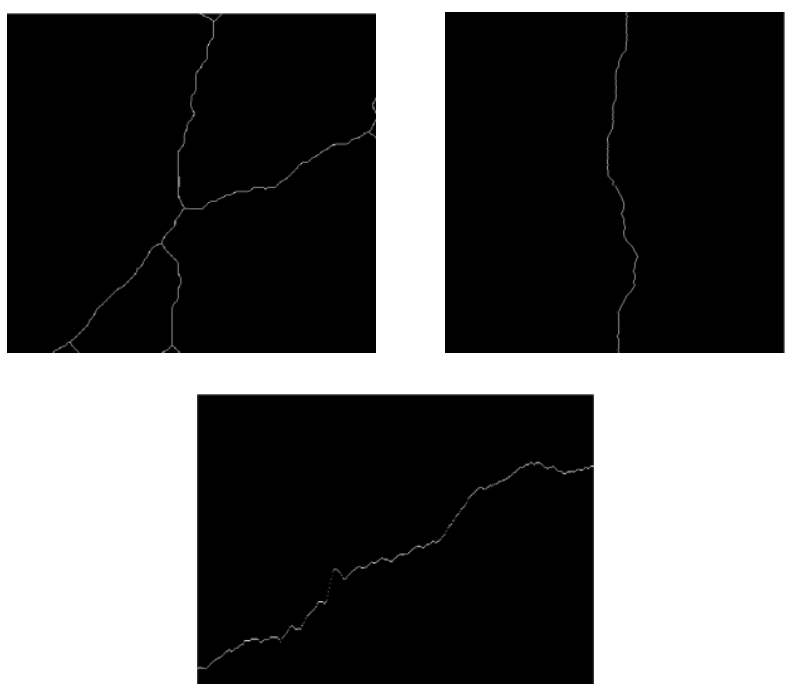

Fig. 9. Skeleton obtained by the improved skeleton algorithm. 

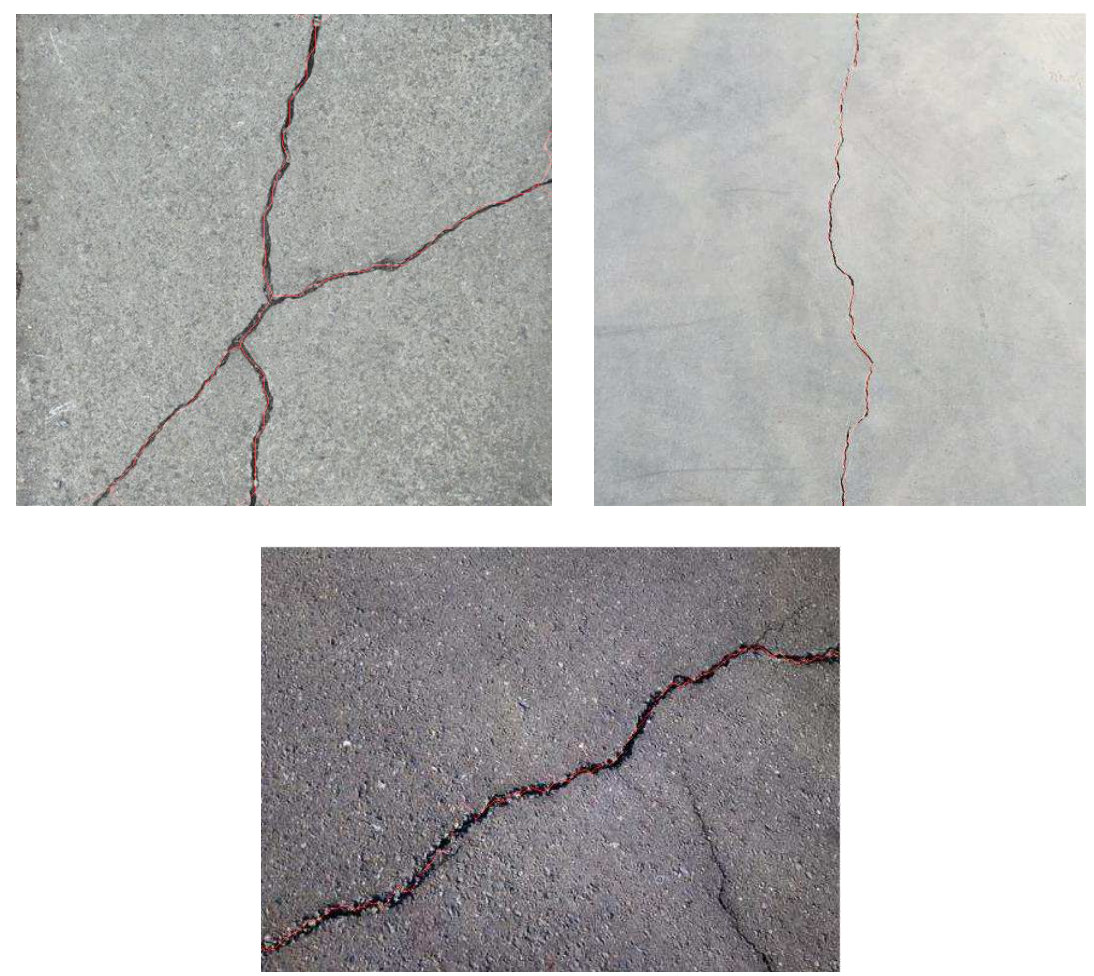

Fig. 10. Crack mark.

\section{Conclusion}

With the development of image processing technology and robotic technology, the requirement of robotic technology for the maintenance of underground sewage pipeline is becoming higher and higher. In this paper, visual-based detection of cement cracks is studied. The main research objects are mesh cracks, fine cracks and dark cracks. Through the comprehensive application of image processing technology, the effective extraction of fracture features is realized. The multi-scale edge feature extraction adopted in this paper can effectively realize the feature extraction of fracture edge. By calculating the area of the maximum connected area, the further de-noising of the fracture image is realized, which is especially suitable for the de-noising of the fracture image. Aiming at the problem that there are many burrs in traditional skeleton algorithm, this paper proposes a new skeleton algorithm and effectively eliminates most of the burrs, which provides corresponding technical support for the subsequent design of crack repair robot. The crack identification method adopted in this paper can basically meet the requirements, but there are still some shortcomings in the identification of reticulated cracks and skeleton extraction, which need further experimental research. 


\section{Acknowledgment:}

This work was supported by grants of National Natural Science Foundation of China This work was supported by grants of National Natural Science Foundation of China (Grant Nos. 51575407, 515505349, 51575338, 51575412, 61733011), the Grants of National Defense Pre-Research Foundation of Wuhan University of Science and Technology (GF201705) and the Open Fund of the Key Laboratory for Metallurgical Equipment and Control of Ministry of Education in Wuhan University of Science and Technology (2018B07) and the DREAM project of EU FP7-ICT (grant no. 611391).

\section{References}

1. Chen F.C., Jahanshahi M.R. NB-CNN: Deep Learning-Based Crack Detection Using Convolutional Neural Network and Naïve Bayes Data Fusion. IEEE Transactions on Industrial Electronics 65(5), 4392-4400 (2018).

2. Hyun-Seok Y., Young-Suk K. Development of a crack recognition algorithm from nonrouted pavement images using artificial neural network and binary logistic regression. KSCE Journal of Civil Engineering 20(4), 1151-1162 (2016).

3. Shu Z., Guo Y. Algorithm on contourlet domain in detection of road cracks for pavement images. Journal of Algorithms and Computational Technology 7(1), 15-26 (2013).

4. Petrik M. Climber-Inspired fuzzy logic approach to crack localization using image analysis. Journal of Advanced Concrete Technology 13(2), 103-111 (2015).

5. Jiang D., Zheng Z., Li G., et al. Gesture recognition based on binocular vision. Cluster Computing (2018). https://doi.org/10.1007/s10586-018-1844-5

6. Li G., Wu H., Jiang G., et al. Dynamic Gesture Recognition in the Internet of Things. IEEE Access 7: 23713-23724 (2019).

7. Nam Y., Choi D.Y., Song B.C. Power-constrained contrast enhancement algorithm using muhiscale retinex for OLED display. IIEEE Transactions on mage Processing 23(8), 3308-3320 (2014).

8. Li G., Jiang D., Zhou Y., et al. Human Lesion Detection Method Based on Image Information and Brain Signal. IEEE Access 7, 11533-11542 (2019).

9. Silverberg J, Yin P. Morphology of Embryonic Epidermis: An Empirical Multiscale Biophysics Approach. Biophysical Journal 114(3):205a (2018).

10. Li H, Song D, Liu Y, et al. Automatic Pavement Crack Detection by Multi-Scale Image Fusion. IEEE Transactions on Intelligent Transportation Systems PP (99):1-12 (2018).

11. Fei Y, Wang K C P, Zhang A, et al. Pixel-Level Cracking Detection on 3D Asphalt Pavement Images Through Deep-Learning-Based CrackNet-V. IEEE Transactions on Intelligent Transportation Systems PP (99):1-12 (2019).

12. Jiang D., Li G., Sun Y., et al. Gesture recognition based on skeletonization algorithm and CNN with ASL database. Multimedia Tools and Applications (2018). https://doi.org/10.1007/s11042-018-6748-0 